Received Date: 05-Jul-2016

Accepted Date: 08-jan-2017

Article Type: Main research

\title{
Labour induction near term for women aged 35 or over: an economic
}

\section{evaluation}

Kate F Walker ${ }^{1}$, Melina Dritsaki ${ }^{2}$, George Bugg ${ }^{3}$, Marion Macpherson ${ }^{3}$, Carol McCormick ${ }^{3}$, Nicky Grace ${ }^{3}$, Chris Wildsmith ${ }^{4}$, Lucy Bradshaw ${ }^{5}$, Gordon CS Smith ${ }^{6}$, James G Thornton ${ }^{1}$

${ }^{1}$ Obstetrics and Gynaecology, Clinical Sciences, University of Nottingham

${ }^{2}$ Oxford Clinical Trials Research Unit, Nuffield Department of Orthopaedics, Rheumatology and Musculoskeletal Sciences, University of Oxford

${ }^{3}$ Obstetrics and Gynaecology, Nottingham University Hospitals NHS Trust

${ }^{4}$ Stillbirth and neonatal death charity (SANDS)

${ }^{5}$ Nottingham Clinical Trials Unit, University of Nottingham

${ }^{6}$ Obstetrics and Gynaecology, NIHR Biomedical Research Centre, University of Cambridge

Corresponding author

Dr Kate F Walker

Maternity Department, Nottingham City Hospital, Nottingham University Hospitals NHS

Trust, Nottingham, NG5 1PB, UK

katefwalker@doctors.org.uk

This article has been accepted for publication and undergone full peer review but has not been through the copyediting, typesetting, pagination and proofreading process, which may lead to differences between this version and the Version of Record. Please cite this article as doi: $10.1111 / 1471-0528.14557$

This article is protected by copyright. All rights reserved. 
Shortened running title: economic evaluation of induction for women aged $\geq 35$ years

\section{Abstract}

Objective: Induction of labour at 39 weeks for nulliparous women aged 35 years and over may prevent stillbirths and does not increase caesarean births, so it may be popular. But the overall costs and benefits of such a policy have not been compared.

Design: A cost-utility analysis alongside a randomised controlled trial (the 35/39 trial).

Setting: Obstetric departments of 38 UK National Health Service hospitals and one UK primary care trust.

Population: Nulliparous women aged 35 years or over on their expected due date, with a singleton live fetus in a cephalic presentation.

Methods: Costs were estimated from the National Health Service and Personal Social Services perspective and quality-adjusted life years (QALYS) were calculated based on patient responses to the EQ-5D at baseline and four weeks.

Main outcome measures: Data on antenatal care, mode of delivery, analgesia in labour, method of induction, EQ-5D (baseline and 4 weeks postnatal) and participant administered postnatal health resource use data was collected.

Results: The intervention was associated with a mean cost saving of $£ 263$ and a small additional gain in QALYs (though not statistically significant), even without considering any possible QALY gains from stillbirth prevention.

Conclusion: A policy of induction of labour at 39 weeks for women of advanced maternal age would save money. 
Keywords: Cost-effectiveness, cost-utility, expectant management, induction of labour, nulliparous, advanced maternal age

Tweetable abstract: A policy of induction of labour at 39 weeks for women of advanced maternal age would save money.

Trial registration: ISRCTN11517275.

\section{Introduction}

The age of childbearing is rising in women living in industrialised nations. Women aged 35 years or over have an increased risk of antepartum stillbirth at term. Induction is currently offered to all women in the UK at 41-42 weeks gestation, when the stillbirth risk is 2 to 3 in $1000(1,2)$; older women experience this risk at earlier gestational ages (2.6 in 1000 from 37 weeks onwards) (3). Labour induction would likely reduce stillbirth, but may also increase caesarean delivery, already high for older women. Although randomised trials of induction for clinical problems near term have not shown an increase in caesarean, no randomised trial of induction based on age had been performed.

The three commonest forms of economic evaluation in health-care are: cost-effectiveness, cost-utility and cost-benefit analyses (4). Uniquely, a cost-utility analysis (CUA) measures outcomes in Quality Adjusted Life Years (QALYS). QALYs can be applied across diseases and specialities allowing policy makers to judge which technologies should be funded (5).

Increasingly economic outcomes for obstetric trials of 'deliver or delay' for various indications are reported. The authors of HYPITAT reported a €831 saving associated with 
induction (6). DIGITAT reported an additional cost of $€ 111$ in the induction group (7). PPROMEXIL reported a $€ 754$ additional cost associated with induction (8). To our knowledge, no cost-utility analyses of an obstetric trial of 'deliver or delay' have been performed.

A multi-centre, randomised [1:1] controlled trial of induction of labour between $39^{0 / 7}$ and $39^{6 / 7}$ weeks gestation or expectant management in 619 nulliparous pregnant women over 35 years of age was performed (ISRCTN11517275). Full clinical results are reported elsewhere (9).

In an intention to treat analysis, there were no significant differences between groups in the proportion of women who had caesarean section (98 (32\%) in the induction group versus $103(33 \%)$ in the expectant group (relative risk [RR] $0.99,95 \% \mathrm{Cl} 0.87-1.14$ ), or instrumental vaginal delivery (115 (38\%) v. 104 (33\%), respectively, RR 1.30, 95\% Cl $0.96-$ 1.77). There were no maternal or infant deaths and no significant differences in maternal experience or adverse maternal or neonatal outcomes. Readmissions (of women) were higher in the control group. The objective of this study was to perform a cost-utility analysis alongside the clinical trial.

\section{Methods}

Trial design

Full details of the $35 / 39$ trial methodology were reported previously (10). Nulliparous women aged 35 years and over on their expected due date, with a singleton live fetus in a cephalic presentation were offered trial entry. Women were randomised at $36^{+0}-39^{+6}$ weeks gestation.

This article is protected by copyright. All rights reserved. 
Intervention

Women were randomly allocated to either induction of labour between $39^{+0}$ and $39^{+6}$ weeks gestation, or to expectant management i.e. awaiting spontaneous onset of labour unless a situation developed necessitating delivery either by induction or caesarean. Women randomised to the expectant management group were offered induction between $41^{+0}$ and $42^{+0}$ (i.e. 7-14 days after the due date), with the exact time determined by their preference and the consultant's usual practice. In all cases where women underwent induction of labour, this was carried out on an inpatient basis.

\section{Outcome measures}

The primary outcome was caesarean delivery and secondary outcomes include instrumental vaginal delivery, intrapartum and postpartum morbidity (need for blood transfusion, systemic infection). The neonatal secondary outcomes were livebirth/stillbirth, birth weight, neonatal intensive care admission, birth trauma and two composite outcomes for serious neonatal morbidity (direct trauma and hypoxic trauma).

Other secondary outcomes included maternal delivery expectation/experience measured by the Childbirth Experience Questionnaire (11) sent at one month postnatal.

\section{Health outcomes}

Health-related quality of life (HRQL) was measured at the time of randomisation and one month post-delivery using the EuroQol EQ-5D-5L measure; responses were used to generate quality-adjusted life-years (QALYs).

This article is protected by copyright. All rights reserved. 
The EQ-5D-5L was launched in 2009 (12) and consisted of 5 levels of response instead of 3 levels as was the case with its predecessor the EQ-5D-3L. A valuation set is currently being developed for the UK for the EQ-5D-5L. At present 'crosswalk' value sets exist for the EQ$5 D-5 L$ whereby values obtained by using the EQ-5D-5L can be used to obtain utility weights for the EQ-5D-3L. A detailed methodology for this is provided in the literature (12).

\section{Collection and valuation of resource use data}

Resource utilisation was captured through two sources: firstly routine health service data collection systems; and secondly patient questionnaires administered at one month postnatal. Full resource use data was collected for all participants from 11th November 2013 (participant number 215 onwards) from the hospital notes at discharge by the research midwife or nurse at the participating centre. Prior to 11th November 2013 incomplete data on resource use was collected for the first 215 participants. From 11th November 2013 all participants received a Health Resource Use Questionnaire at one month postnatal to capture resource use after hospital discharge. Data on resource use after hospital discharge was not collected for the first 215 participants.

The economic assessment method as far as possible adhered to the recommendations of the NICE Reference Case(13). Primary research methods were followed to estimate the costs of the treatment options, including drugs and rehabilitation inputs.

Unit costs for health and social care resources were derived from local and national sources and estimated in line with best practice (14-18). Primary research using established accounting methods were also required to estimate unit costs. Costs were standardised to 
current prices where possible. Units costs fell into 5 main groups: staff, procedure related, investigations, admissions and drugs (used for the process of induction of labour/analgesia in labour/other). Unit costs of health and social care resource items are shown in Tables S1.

\section{Cost Effectiveness Analysis}

A prospective economic evaluation, conducted from a NHS and personal social services (PSS) perspective, was integrated into the trial design. The economic evaluation estimated the difference in the cost of resource inputs used by participants in the two arms of the trial, allowing comparisons to be made between the two treatment options (induction of labour versus expectant management) for nulliparous women over 35 years of age and enabling costs and consequences to be compared.

Cost-utility analysis assesses two alternative courses of action in terms of their cost and outcome expressed in QALYs. The comparison is expressed using the Incremental Cost Effectiveness Ratio (ICER). The ICER is a measure of the additional cost per additional unit of health gain produced by one course of action compared to another, i.e. the cost per QALY gained. The cost effectiveness threshold is described as what society is willing to pay for an additional unit of health gain (QALY). The threshold currently set by NICE is $£ 20,000$ $£ 30,000$ (13). We also presented the results in terms of Incremental Net Benefit (INB) statistics, calculated by multiplying the incremental effects by an assumed monetary value of a QALY (the cost effectiveness threshold) and subtracting the incremental cost. We calculated INB statistics based on $£ 10,000$ to $£ 50,000$ per QALY. A positive INB suggests that the intervention is cost-effective compared with usual care at the defined threshold. Decision uncertainty was addressed by constructing cost-effectiveness acceptability curves across cost-effectiveness threshold values of between $£ 0$ and $£ 100,000$ for the outcomes of 
interest. If the figure is greater than 0.5 , it indicates that the intervention is more likely to be cost-effective than not.

Using an intention to treat approach, costs and outcomes for each trial participant were calculated. The costs and outcomes for the two groups were analysed using Stata Version 13. Non-parametric bootstrap estimation was used to derive $95 \%$ confidence intervals for mean cost differences between the trial groups.

The base case assumption was that induction neither prevented nor caused stillbirth. We performed a sensitivity analysis to measure the cost effectiveness of induction for a range of stillbirth rate prevented/caused.

Non-parametric estimation is required as health care costs are typically positively skewed i.e. a small number of patients will incur very high costs. Bootstrap estimation allows a statistic of interest (such as the mean) to be calculated from samples which are not normally distributed, or indeed samples where the distribution is unknown. Our results were based on 1000 bootstrap samples, which was sufficient to provide estimated costs and effects.

\section{Results}

Full resource use data at hospital discharge was collected from the hospital records for 380 (61\%) trial participants from $11^{\text {th }}$ February 2013 onwards (participants $239-619$ ). Data on antenatal care, mode of delivery, analgesia required in labour, method of induction of labour used was available for all 619 participants. Of the 380 trial participants for whom economic outcomes were collected: EQ-5D at baseline was available for 349 (92\%) 
participants; EQ-5D at 4 weeks postnatal was available for 277 (73\%) participants; health resource use after hospital discharge data was available for 297 (78\%) participants. The economic analysis was performed on the data for 380 trial participants. Relevant unit costs are presented in Table S1.

There were two predominant differences in resource use between the induction of labour and expectant management groups (Table S2). The first was a higher mean cost for assisted delivery in the induction group than the expectant group (mean cost 1006 vs. 888) which was offset by a lower mean cost for normal vaginal delivery in the induction group than the expectant group (mean cost 585 vs. 672). The second was a higher mean cost for hospital readmission in the expectant group (due to three more readmissions) than the induction group (mean cost 383 vs. 128), this was the single highest mean difference in cost for an individual cost category between the two groups.

Healthcare cost data tends to be highly skewed. This can only be partly addressed using parametric methods because the arithmetic mean is the informative instrument, providing information about the cost of treating all patients, which is required for healthcare policy decisions (19). In order to fully address the skewed nature of the data we performed additional non-parametric analyses on the cost differences between the two groups. Initially, we conducted a bootstrap (using 1,000 replications with resampling) of the mean cost differences for each cost category within this dataset, for women in the induction group and the expectant group (Table S2).

This article is protected by copyright. All rights reserved. 
These resource quantities were multiplied by the relevant unit costs (Table S1) to provide estimates of the means costs per patient (Table S2). Differences between the groups in the cost of healthcare use show a mean cost saving of $£ 263$ (95\% confidence interval [CI] (-£646 to $f 174$ ) using non-parametric bootstrap estimation) associated with induction of labour, though there was a wide confidence interval around this estimate. This difference was largely attributable to the higher mean cost of readmissions in the expectant group than the induction group.

Mean EQ-5D utility scores for women in the induction of labour group and expectant management group at baseline and at 4 weeks postnatal were used to calculate the mean QALYs gained in each group. The mean QALYs gained over the trial period based on EQ-5D utility measure were slightly higher for the induction of labour group than the expectant management group (0.03 versus 0.01$)$ (Table 1) although this was not statistically significant.

The results of the incremental cost-effectiveness analysis of the induction of labour group compared with expectant management group are presented in Table 2. ICER was calculated using differences in costs divided by differences in effects between induction of labour and using the expectant management group as the reference group. As the intervention (induction of labour) is associated with a gain in QALYs and a lower mean cost than the expectant management group this results in a negative ICER $(-£ 114,526)$, reflecting both cost-savings and positive QALYs. The differences in effects are very small; however these are magnified in the ICER calculations, as the mean differences in effects are used as the denominators of the ICER statistics. These estimates have very wide confidence intervals. 
One thousand bootstrapped weighted estimates for each of the ICERs are presented on cost-effectiveness planes (Figure 2).

A weighted bootstrapped scatterplot is represented graphically in cost-effectiveness planes in Figure 1. The origin of the cost-effectiveness plane represents the average cost and average effect for the reference group, in this case expectant management. The point estimate of mean ICER therefore represents the incremental changes in costs and effects generated by the differences between the induction of labour and expectant group. In each analysis, 1,000 bootstrapped mean ICERs were plotted on the cost effectiveness plane. They show the uncertainty around the mean reported ICERs.

Cost-effectiveness acceptability curves (CEACs) were generated to show the probability of the induction of labour planning being optimal in terms of cost-effectiveness at alternative cost-effectiveness thresholds held by decision-makers. Cost-effectiveness thresholds were varied from $£ 10,000$ to $£ 50,000$, with $£ 20000$ considered to be the most intuitive threshold for the QALYs. Induction of labour plan has a $100 \%$ probability of being cost effective comparing to the expectant management plan in nulliparous pregnant women over 35 years age across all cost-effectiveness thresholds.

\section{Discussion}

Main findings

Induction of labour at 39 weeks for women of advanced maternal age is associated with a small gain in QALYs and is cheaper by $f 263$ on average than expectant management. The difference in cost between the two arms of the study predominantly arose due to an 
increase in postnatal readmissions to hospital (three more) in the expectant management arm of the study.

\section{Strengths and limitations}

The use of a prospective randomised trial design provided unbiased and comprehensive data to perform a cost-utility analysis. Unfortunately complete health resource use data was only captured from November 2013 onwards. This means the cost utility analysis could only be performed for 380 (61\%) participants.

There has been a recent increase in reporting of economic outcomes for obstetric trials of 'deliver or delay' for various indications with some reporting a cost saving associated with induction $(6)$ and others a cost incurred $(7,8)$. Previous economic evaluations have taken the form of cost-effectiveness analyses. This is the first cost-utility analysis of an obstetric trial of 'deliver or delay'. Allowing the outcomes to be measured in QALYs means the value of the intervention can be considered in a broader context by policy makers. Previous economic evaluations have collected data on resource use until hospital discharge (DIGITAT, PPROMEXIL) and will therefore have missed costs incurred in the postnatal period which had a big impact on the results of our study.

The limited time horizon of the study meant that the follow up of outcomes were limited to up to 4 week-postnatal care. It is frequently observed that morbidities associated with labour and birth and its management affect women and babies in the long run. Follow up over weeks or longer to monitor recovery, or a future assessment of the outcomes for mothers and babies at a later date, would shed more light on long term cost-effectiveness. 
The analysis presented here assumes that induction neither prevents nor causes stillbirth. If plausibly we assume that induction prevents say half of all antepartum stillbirths from 39 weeks onwards in this group of women, and an absolute rate of 1 in 500 for such stillbirths beyond 39 weeks, then a policy of induction should result in 1 in 1000 women having a stillbirth prevented. If stillbirth is associated with a loss of 25 QALYs (20), then the prevention of 1 in 1000 women having a stillbirth would result in the addition of 25 QALYs per 1000 women or 0.025 QALY per woman. This hypothetical, but plausible gain is ten times larger than the net increase in QALY per woman from other aspects of care. Its inclusion would result in an even more cost effective intervention.

While the cost of the induction may not be higher than expectant management, the authors acknowledge that were induction to be offered to all women of advanced maternal age this would have an impact on the already stretched working capacity of maternity units.

\section{Interpretation}

Induction of labour at 39 weeks for women of advanced maternal age has no adverse effects on short term maternal or neonatal outcomes, in particular it does not increase caesareans. It therefore appears safe to be tested as a strategy to prevent late antepartum stillbirths in this group of women. This cost-utility analysis has shown that should such a strategy be adopted it would likely save money even without preventing stillbirths.

This article is protected by copyright. All rights reserved. 


\section{Conclusion}

This cost-utility analysis has shown that a policy of induction of labour at 39 weeks for women of advanced maternal age would probably save money even if it did not prevent stillbirths.

\section{Acknowledgements}

None

\section{Disclosure of Interests}

Dr. Smith reports receiving fees for serving on an advisory board from Roche Diagnostics, consulting fees from GlaxoSmith-Kline, equipment loans from Roche Diagnostics and General Electric, travel support from Roche Diagnostics and Chiesi, and grant support from GlaxoSmithKline and Action Medical Research, and being named as an inventor on a pending patent (PCT/EP2014/062602) filed by GlaxoSmithKline related to retosiban as a preventive treatment for preterm labour in women with increased uterine stretch. No other potential conflict of interest relevant to this article was reported. The ICMJE disclosure forms are available as online supporting information.

\section{Contribution to Authorship}

KW, MD and JGT designed the study. LB advised on the statistical analysis of the study. KW, GB, CM, MM, CW, NG, GS and JGT conducted the study. KW and MD analysed the data. KW wrote the paper and prepared the figures and tables. All the authors revised the paper and agreed to the submission of the final version of the manuscript. 
Details of Ethics Approval

The study received ethical approval from the Derby 1 Research Ethics Committee (NRES 12/EM/0003) on the $12^{\text {th }}$ January 2012.

Funding

Supported by a grant (PB-PG-0610-22275) from the Research for Patient Benefit Programme of the National Institute for Health Research.

\section{References}

1. Gulmezoglu AM, Crowther CA, Middleton P. Induction of labour for improving birth outcomes for women at or beyond term. Cochrane Database of Systematic Reviews. 2006(4).

2. Health NCCfWsaCs. Induction of labour. London, UK: National Institue for Health and Clinical Excellence; 2008.

3. Reddy UM, Ko CW, Willinger M. Maternal age and the risk of stillbirth throughout pregnancy in the United States. Am J Obstet Gynecol. 2006;195(3):76470.

4. Fox-Rushby J CJ. Economic Evaluation. R BNaR, editor. Berkshire: Open University Press; 2005.

5. Sassi F. Calculating QALYs, comparing QALY and DALY calculations. Health Policy Plann. 2006;21(5):402-8.

6. Vijgen SMC, Koopmans CM, Opmeer BC, Groen H, Bijlenga D, Aarnoudse $\mathrm{JG}$, et al. An economic analysis of induction of labour and expectant monitoring in women with gestational hypertension or pre-eclampsia at term (HYPITAT trial). BjogInt J Obstet Gy. 2010;117(13):1577-85.

7. Vijgen SMC, Boers KE, Opmeer BC, Bijlenga D, Bekedam DJ, Bloemenkamp $\mathrm{KWM}$, et al. Economic analysis comparing induction of labour and expectant management for intrauterine, growth restriction at term (DIGITAT trial). Eur J Obstet Gyn R B. 2013;170(2):358-63.

8. Vijgen SMC, Van der Ham DP, Bijlenga D, Van Beek JJ, Bloemenkamp KWM, Kwee A, et al. Economic analysis comparing induction of labor and expectant management in women with preterm prelabor rupture of membranes between 34 and 37 weeks (PPROMEXIL trial). Acta Obstet Gyn Scan. 2014;93(4):374-81.

9. Walker KF, Bugg GJ, Macpherson M, McCormick C, Grace N, Wildsmith C, et al. Randomized Trial of Labor Induction in Women 35 Years of Age or Older. N Engl J Med. 2016;374(9):813-22.

10. Walker KF, Bugg G, Macpherson M, McCormick C, Wildsmith C, Smith G, et al. Induction of labour versus expectant management for nulliparous women over 35 
years of age: a multi-centre prospective, randomised controlled trial. Bmc Pregnancy Childb. 2012;12.

11. Dencker A, Taft C, Bergqvist L, Lilja H, Berg M. Childbirth experience questionnaire (CEQ): development and evaluation of a multidimensional instrument. Bmc Pregnancy Childb. 2010;10.

12. Herdman M, Gudex C, Lloyd A, Janssen MF, Kind P, Parkin D, et al. Development and preliminary testing of the new five-level version of EQ-5D (EQ-5D5L). Qual Life Res. 2011;20(10):1727-36.

13. Excellence NlfHaC. Guide to the methods of technology appraisal. 2008.

14. Unit PSSR. Unit Costs of Health and Social Care 2013. University of Kent: Personal Social Services Research Unit; 2013.

15. Committee JF. British National Formulary. London: British Medical Association and Royal Pharmaceutical Society of Great Britain; 2013 October February 2014.

16. National Schedule of Reference Costs Year 2012-2013 Department of Health; 2013.

17. Catalogue NSCO. NHS Supply Chain Online Catalogue In: Service NH, editor. 2014.

18. Schroeder L PS, Patel N, Hollowell J, Puddicombe D, Redshaw M, et al. Birthplace cost-effectiveness analysis of planned place of birth: individual level analysis.; 2011.

19. Thompson SG, Barber JA. How should cost data in pragmatic randomised trials be analysed? Brit Med J. 2000;320(7243):1197-200.

20. Women's NCCf, Health aCs. Antenatal care: routine care for the healthy pregnant woman. London, UK: RCOG Press; 2008. 
Figure 1 Cost effectiveness plane: induction plan compared to expectant management

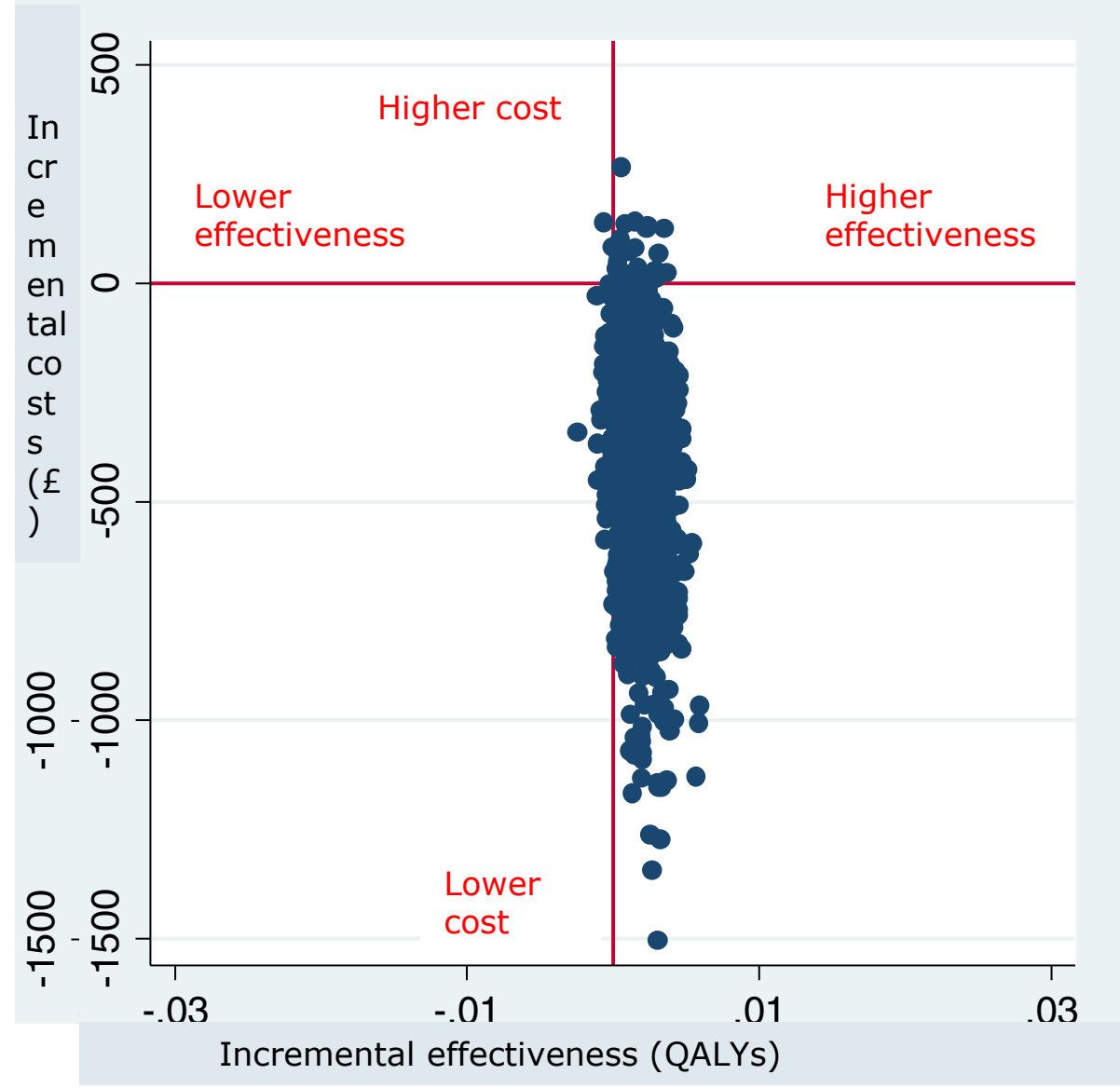

This article is protected by copyright. All rights reserved. 
Figure 2. Cost effectiveness acceptability curves for induction plan compared to expectant management

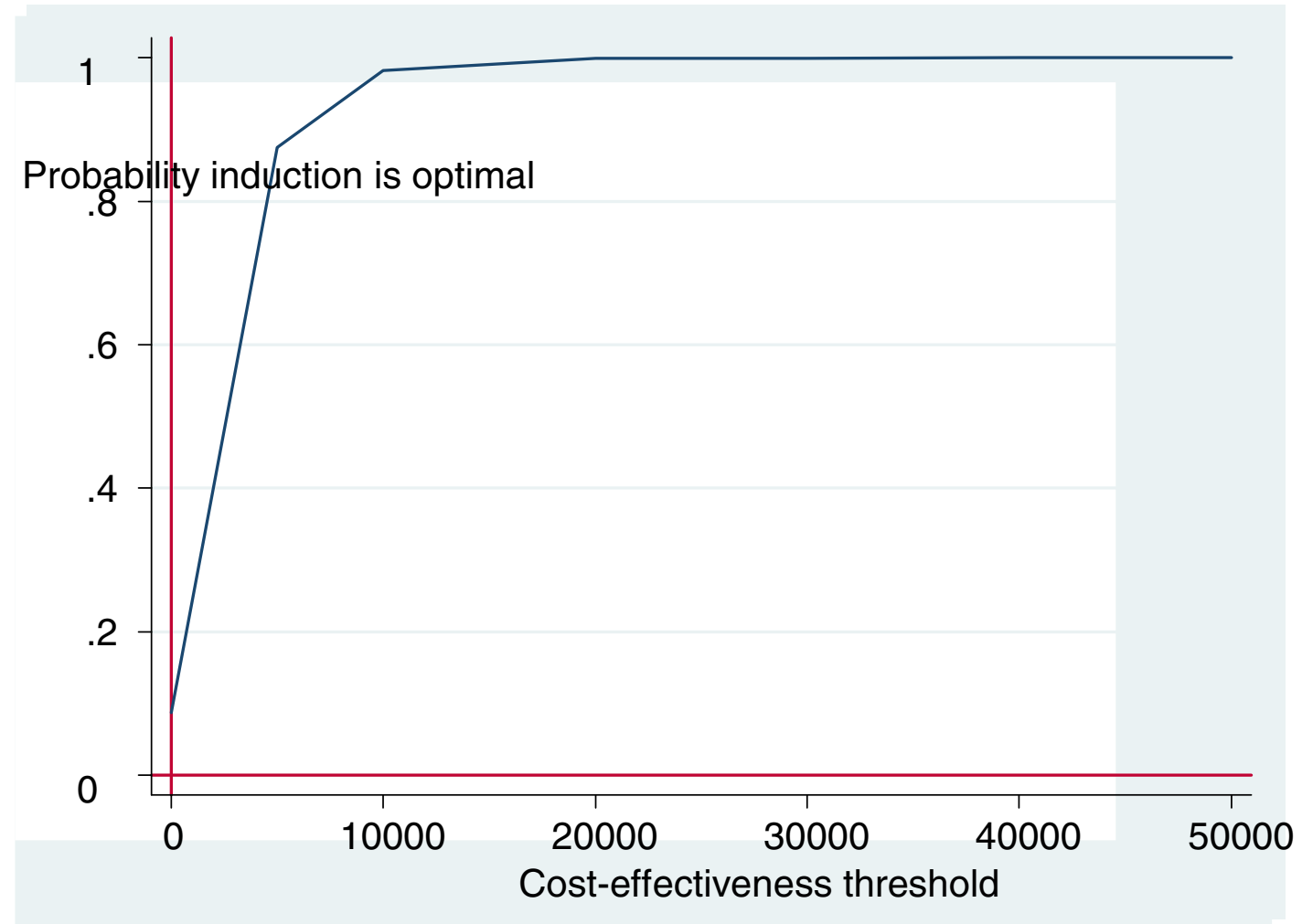

The CEAC illustrates the probability of induction being more cost-effective than expectant management at different thresholds of decision makers' willingness to pay for a QALY.

This article is protected by copyright. All rights reserved. 